\title{
Medición De La Fluidez En La Escritura Colaborativa E Individual En EI Idioma Inglés
}

\author{
Angel Paúl Obregón Mayorga, Mgs \\ Jazmina Ivonne Mena Mayorga, Mgs \\ Ana Gabriela Reinoso Espinosa, Mgs \\ Nelly Margarita Padilla Padilla, Mgs
}

Magíster en la Enseñanza del Idioma Inglés Como Lengua Extranjera,

Docente de la Escuela Superior Politécnica de Chimborazo,

Centro de Idiomas, Ecuador

Doi:10.19044/esj.2019.v15n5p39 ～$\quad$ URL:http://dx.doi.org/10.19044/esj.2019.v15n5p39

\section{Resumen}

El propósito de esta investigación es determinar si la fluidez en la escritura colaborativa, teniendo en cuenta el número total de palabras escritas, es mayor al número total de palabras escritas individualmente. El método empleado para este finmaneja datos cuantitativos. Los participantes en esta investigación fueron 33 estudiantes del tercer nivel de inglés de una universidad pública en Ecuador. Para recopilar los datos se utilizaron como instrumentos los ensayos escritos por los estudiantes además de un cuestionario con preguntas cerradas para medir el nivel de satisfacción al utilizar Google Docs como herramienta de escritura colaborativa. Los resultados de las mediciones mostraron que había un mayor número de palabras, cláusulas y oraciones en los ensayos colaborativos en comparación con los ensayos escritos individualmente. De la misma manera, los resultados del cuestionario mostraron que la mayoría de los estudiantes dijeron tener beneficios positivos al usar esta herramienta colaborativa.

Palabras claves: Medición; ensayo colaborativo; fluidez; cláusulas 


\title{
Measurement of Fluency in Collaborative and Individual Writing in English Language
}

\author{
Angel Paúl Obregón Mayorga, Mgs \\ Jazmina Ivonne Mena Mayorga, Mgs \\ Ana Gabriela Reinoso Espinosa, Mgs \\ Nelly Margarita Padilla Padilla, Mgs \\ Magíster en la Enseñanza del Idioma Inglés Como Lengua Extranjera, \\ Docente de la Escuela Superior Politécnica de Chimborazo, \\ Centro de Idiomas, Ecuador
}

\begin{abstract}
This paper focuses on determining whether the fluency in collaborative writing, taking into account the total number of written words, is greater than the total number of words written individually. The study method used a quantitative data analysis. The participants in this research were 33 students of the third level of English of a public university in Ecuador. The instruments used to collect the data were the essays written by the students, as well as a questionnaire with closed questions. The level of satisfaction was measured using Google Docs as a collaborative writing tool. The results of the measurements showed that there were a significant number of words, clauses, and sentences in the collaborative trials when compared to the essays written individually. In the same way, the results of the questionnaire showed that most of the students stated that the use of collaborative tool has a significant positive impact on them.
\end{abstract}

Keywords: Measurement, collaborative essay, fluency, clauses

\section{Introducción}

Las nuevas herramientas tecnológicas hoy en día son de vital importancia, especialmente en el proceso de aprendizaje de la escritura en inglés. Estudios anteriores tales como el de Storch en el año 2011 muestran que la escritura colaborativa brinda muchos beneficios a los estudiantes y podría ayudar a mejorar las habilidades de escritura, así como también mejorar el aprendizaje del idioma inglés. Sin embargo, el uso de actividades de escritura colaborativa en las clases de inglés como segunda lengua es muy limitado (Storch, 2011). 
La Guía Nacional de Currículo en inglés (2012) menciona que los estudiantes universitarios deberían haber alcanzado un nivel de inglés de dominio B1 de acuerdo con el Marco Común Europeo de Referencia para las Lenguas (MCER), lo que implicaría que los estudiantes cuentan con aptitudes para comunicarse utilizando un inglés básico. No obstante, en la mayoría de las universidades a los estudiantes les resulta difícil escribir y generar ideas por sí mismos, ya que no les gusta escribir en inglés por distintas razones. Por tal motivo es importante que los profesores de inglés implementen actividades de escritura colaborativa en sus clases para motivar a los estudiantes a que escriban más en un ambiente donde todos se sientan cómodos.

En lo que respecta a la fluidez, esta es un indicador del dominio de la lengua extranjera. Para este estudio se ha considerado la medición de la fluidez de la escritura basada en Larsen-Freeman (1978) y Henry (1996), quienes toman el número promedio de palabras por composición de estudiantes de inglés como su segunda lengua. Según Larsen-Freeman (1978), hay un aumento en el número de palabras por composición a medida que aumenta el nivel de grupo.

Otro método citado en esta investigación fue el propuesto por (WolfeQuintero, Inagaki, \& kim, 1998), el cual mide la longitud de unidad de produccion. Así, la longitud de la unidad de producción se basa en la medida de los siguientes valores: la media de la longitud de la clausulas (MLC), la media de longitud de las oraciones (MLS) y la media de la longitud de la unidad T(MLT), donde MLC es igual al número de palabras dividido entre el número de cláusulas, MLS es igual al número de palabras dividido entre el número de oraciones y MLT es igual al número de palabras dividido entre el número de unidades $\mathrm{T}$.

El propósito de esta investigación fue verificar que a través de la escritura colaborativa se produce más texto que a través de la escritura individual, así como también confirmar los beneficios al usar una herramienta colaborativa. Sin embargo, es importante mencionar que los resultados en este estudio reflejan solamente el nivel de los estudiantes que participaron en esta investigación; por consiguiente, los resultados emitidos en este muestreo no se pueden generalizar, siendo esta una limitación junto con el nivel de inglés de cada estudiante y los conocimientos de computación que fueron variados.

Este trabajo respondió a las siguientes preguntas de investigación:

¿Producen más texto las actividades de escritura colaborativa que las actividades de escritura individuales?

¿Existen beneficios al utilizar escritura colaborativa?

\section{Metodología}

Como primer paso para formar los grupos para las actividades de escritura, los estudiantes rindieron una prueba de ubicación en donde se midió 
el dominio de la escritura del idioma inglés. Esta prueba midió el nivel de escritura contando el número de palabras producidas por los estudiantes, método sugerido por (Larsen-Freeman, 1978). Los participantes fueron 33 estudiantes del tercer nivel de inglés, de los cuales 22 fueron hombres y 11 mujeres. Los estudiantes tuvieron 30 minutos para escribir un ensayo con el tema ¿Por qué el inglés es importante en su carrera elegida? Una vez terminada la prueba los estudiantes fueron ubicados en dos categorías en base a los resultados que obtuvieron en la prueba escrita de ubicación; los estudiantes con una producción de más de 150 palabras fueron asignados a la categoría intermedio, mientras que los estudiantes con una producción menor a 150 palabras fueron asignados a la categoría principiantes.

Se seleccionó un estudiante de cada categoría hasta formar seis grupos de cinco y seis estudiantes. Los grupos se clasificaron de la siguiente manera: Grupo A, seis estudiantes, Grupo B cinco estudiantes, Grupo C seis estudiantes, Grupo D cinco estudiantes, Grupo E cinco estudiantes, Grupo F seis estudiantes.

Tres grupos fueron seleccionados para participar en las actividades de escritura individual, mientras que la otra mitad participó en las actividades de escritura colaborativa. Cada semana, cada grupo cambiaba su actividad de escritura colaborativa a individual y viceversa y así sucesivamente hasta completar el proceso de escritura de cuatro semanas.

Los estudiantes utilizaron Google Docs para escribir tanto colaborativa como individualmente. En las dos actividades, los estudiantes escribieron un ensayo en inglés sobre un tema en particular asignado por el profesor. A cada grupo se le dio un tiempo de treinta minutos para la planificación y el intercambio de ideas, treinta minutos para escribir un borrador y treinta minutos para revisar el trabajo.

Los dos grupos de estudiantes se reunieron tres días por semana durante un mes y pasaron dos horas cada día escribiendo sus composiciones asignadas. Para medir la fluidez de escritura por ensayo este estudio considero el método propuesto por (Larsen-Freeman, 1978; Henry, 1996), así como también se consideró el método que mide la longitud de unidad de producción sugerido por Wolfe-Quintero et al. (1998).

De acuerdo a (Stein, Bernas \& Calicchia, 1997) los estudiantes se vuelven más conscientes de su proceso de aprendizaje durante una actividad de escritura colaborativa; esto se debe a que generan una fuente de conocimiento durante la planificación, discusión y negociación de ideas.

La longitud de unidad de producción de cada ensayo fue medida utilizando el software "Automatic analysis of syntactic complexity in second language writing" creado por (Lu, 2010). Esta aplicación web fue muy práctica ya que permitió analizar la complejidad sintáctica de escribir muestras en inglés de hasta cincuenta archivos a la vez. 
Tabla 1

\begin{tabular}{|c|c|c|c|c|c|c|c|}
\hline \multicolumn{8}{|c|}{ Longitud de Producción Grupo A } \\
\hline Ensayo & $\begin{array}{c}\text { No. de } \\
\text { Palabras }\end{array}$ & $\mathbf{S}$ & C & $\mathbf{T}$ & MLS & MLT & MLC \\
\hline \multicolumn{8}{|c|}{ Producción Colaborativa $(N=6)$} \\
\hline 1 & 330 & 19 & 31 & 21 & 17.37 & 15.71 & 10.65 \\
\hline 2 & 709 & 32 & 72 & 35 & 22.16 & 20.26 & 9.85 \\
\hline 3 & 841 & 30 & 61 & 28 & 28.03 & 30.04 & 13.79 \\
\hline 4 & 722 & 27 & 82 & 33 & 26.74 & 21.88 & 8.80 \\
\hline 5 & 1055 & 49 & 105 & 59 & 21.53 & 17.88 & 10.05 \\
\hline 6 & 1229 & 34 & 120 & 46 & 36.15 & 26.72 & 10.24 \\
\hline MEDIA & 814.33 & 31.83 & 78.5 & 37 & 25.33 & 22.08 & 10.56 \\
\hline MEDIANA & 781.5 & 31 & 77 & 34 & 24.45 & 21.07 & 10.14 \\
\hline MODA & N/A & N/A & N/A & N/A & N/A & N/A & N/A \\
\hline $\mathrm{DE}$ & 311.38 & 9.91 & 31.75 & 13.58 & 6.55 & 5.41 & 1.70 \\
\hline \multicolumn{8}{|c|}{ Producción Individual $(N=6)$} \\
\hline 1 & 267 & 6 & 37 & 10 & 44.50 & 26.70 & 7.22 \\
\hline 2 & 131 & 5 & 8 & 4 & 26.20 & 32.75 & 16.38 \\
\hline 3 & 199 & 13 & 28 & 15 & 15.31 & 13.27 & 7.11 \\
\hline 4 & 210 & 11 & 28 & 11 & 19.09 & 19.09 & 7.50 \\
\hline 5 & 224 & 10 & 25 & 11 & 22.40 & 20.36 & 8.96 \\
\hline 6 & 337 & 6 & 29 & 9 & 56.17 & 37.44 & 11.62 \\
\hline MEDIA & 228 & 8.5 & 25.83 & 10 & 30.61 & 24.94 & 9.80 \\
\hline MEDIANA & 217 & 8 & 28 & 10.5 & 24.3 & 23.53 & 8.23 \\
\hline MODA & N/A & 6 & 28 & 11 & N/A & N/A & N/A \\
\hline $\mathrm{DE}$ & 69.27 & 3.27 & 9.62 & 3.58 & 16.12 & 9.08 & 3.65 \\
\hline
\end{tabular}

Nota: $S=$ oraciones, $C=$ cláusulas, $T=$ t-unidades, $M L S=$ palabras por oración, $M L T=$ palabras por unidad T, $M L C=$ palabras por cláusula, $D E=$ desviación estándar, $N=$ tamaño de la muestra (ensayos)

Como se muestra en la Tabla 1 los ensayos producidos en colaboración fueron más fluidos con respecto al número total de palabras, oraciones, cláusulas, unidades $\mathrm{T}$ y palabras por cláusula (MLC) que los ensayos producidos individualmente. Sin embargo, las palabras por unidad T (MLT) y las palabras por oración (MLS) fueron ligeramente más largas en los ensayos producidos individualmente. De acuerdo a Ortega (2003) y Henry (1996) las palabras por oración, palabras por cláusula y palabras por unidades $\mathrm{T}$ son usadas para medir la complejidad sintáctica. Esto significa que los estudiantes que trabajaron como parte de un grupo colaborativo crearon ensayos más largos y complejos que los estudiantes que escribieron individualmente; esto se debe a que los estudiantes compartieron ideas a través de la negociación, 
discusión y planificación con los miembros de su equipo como mencionan Stein et al. (1997).

Tabla 2

\begin{tabular}{|c|c|c|c|c|c|c|c|}
\hline \multicolumn{8}{|c|}{ Longitud de Producción Grupo B } \\
\hline Ensayo & $\begin{array}{l}\text { No. de } \\
\text { Palabras }\end{array}$ & S & $\mathrm{C}$ & $\mathrm{T}$ & MLS & MLT & MLC \\
\hline \multicolumn{8}{|c|}{ Producción Colaborativa $(N=6)$} \\
\hline 1 & 582 & 32 & 58 & 34 & 18.188 & 17.118 & 10.035 \\
\hline 2 & 403 & 11 & 20 & 10 & 36.636 & 40.300 & 20.150 \\
\hline 3 & 530 & 25 & 44 & 23 & 21.200 & 23.044 & 12.046 \\
\hline 4 & 812 & 28 & 63 & 28 & 29.000 & 29.000 & 12.889 \\
\hline 5 & 504 & 23 & 36 & 22 & 21.913 & 22.909 & 14.000 \\
\hline 6 & 497 & 32 & 37 & 30 & 15.531 & 16.567 & 13.432 \\
\hline MEDIA & 554.667 & 25.167 & 43.000 & 24.500 & 23.745 & 24.823 & 13.759 \\
\hline MEDIANA & 517.000 & 26.500 & 40.500 & 25.500 & 21.557 & 22.976 & 13.161 \\
\hline MODA & N/A & 32.000 & N/A & N/A & N/A & N/A & N/A \\
\hline $\mathrm{DE}$ & 138.904 & 7.834 & 15.748 & 8.385 & 7.772 & 8.847 & 3.423 \\
\hline \multicolumn{8}{|c|}{ Producción Individual $(N=6)$} \\
\hline 1 & 184 & 17 & 19 & 13 & 10.824 & 14.154 & 9.684 \\
\hline 2 & 509 & 12 & 46 & 17 & 42.417 & 29.941 & 11.065 \\
\hline 3 & 169 & 8 & 19 & 9 & 21.125 & 18.778 & 8.895 \\
\hline 4 & 212 & 9 & 25 & 8 & 23.556 & 26.500 & 8.480 \\
\hline 5 & 252 & 33 & 37 & 33 & 7.636 & 7.636 & 6.811 \\
\hline 6 & 211 & 24 & 23 & 20 & 8.792 & 10.550 & 9.174 \\
\hline MEDIA & 256.167 & 17.167 & 28.167 & 16.667 & 19.058 & 17.927 & 9.018 \\
\hline MEDIANA & 211.500 & 14.500 & 24.000 & 15.000 & 15.974 & 16.466 & 9.034 \\
\hline MODA & N/A & N/A & 19.000 & N/A & N/A & N/A & N/A \\
\hline $\mathrm{DE}$ & 127.074 & 9.745 & 10.962 & 9.223 & 13.220 & 8.867 & 1.403 \\
\hline
\end{tabular}

Nota: $S=$ oraciones, $C=$ cláusulas, $T=$ t-unidades, $M L S=$ palabras por oración, $M L T=$ palabras por unidad T, $M L C=$ palabras por cláusula, $D E=$ desviación estándar, $N=$ tamaño de la muestra (ensayos)

Como se muestra en la Tabla 2, los ensayos producidos en colaboración fueron más fluidos con respecto al número total de palabras, oraciones, cláusulas, unidades T, palabras por oración (MLS), palabras por cláusula (MLC), palabras por unidad T (MLT) que los ensayos escritos individualmente. Los estudiantes que trabajaron como parte de un grupo colaborativo crearon ensayos más largos que los estudiantes que escribieron individualmente, esto se debe a que los estudiantes intercambiaron ideas a través de la negociación, discusión y planificación con los miembros de su equipo ratificando lo que mencionan Stein et al. (1997).

Las palabras por oración (MLS), las palabras por cláusula (MLC) y las palabras por unidad T (MLT) también son más largas en los ensayos de 
colaboración, lo cual corrobora lo que Wolfe-Quintero et al. (1998), mencionó con respecto a la medida de la longitud de unidad de producción.

\section{Tabla 3}

\begin{tabular}{|c|c|c|c|c|c|c|c|}
\hline \multicolumn{8}{|c|}{ Longitud de Producción Grupo C } \\
\hline Ensayo & $\begin{array}{c}\text { No. de } \\
\text { Palabras }\end{array}$ & S & $\mathrm{C}$ & $\mathrm{T}$ & MLS & MLT & MLC \\
\hline \multicolumn{8}{|c|}{ Producción Colaborativa $(N=6)$} \\
\hline 1 & 756 & 28 & 71 & 23 & 27.00 & 32.87 & 10.65 \\
\hline 2 & 491 & 24 & 49 & 28 & 20.46 & 17.54 & 10.02 \\
\hline 3 & 777 & 34 & 70 & 35 & 22.85 & 22.20 & 11.10 \\
\hline 4 & 469 & 19 & 44 & 19 & 24.68 & 24.68 & 10.66 \\
\hline 5 & 696 & 26 & 66 & 28 & 26.77 & 24.86 & 10.55 \\
\hline 6 & 834 & 33 & 87 & 36 & 25.27 & 23.17 & 9.59 \\
\hline MEDIA & 670.5 & 27.33 & 64.50 & 28.17 & 24.51 & 24.22 & 10.43 \\
\hline MEDIANA & 726 & 27 & 68 & 28 & 24.98 & 23.93 & 10.60 \\
\hline MODA & N/A & N/A & N/A & 28 & N/A & N/A & N/A \\
\hline $\mathrm{DE}$ & 154.18 & 5.65 & 15.76 & 6.62 & 2.49 & 5.01 & 0.54 \\
\hline \multicolumn{8}{|c|}{ Producción Individual $(N=6)$} \\
\hline 1 & 289 & 13 & 55 & 12 & 22.23 & 24.08 & 7.61 \\
\hline 2 & 365 & 19 & 42 & 22 & 19.21 & 16.59 & 8.69 \\
\hline 3 & 318 & 10 & 21 & 9 & 31.80 & 35.33 & 15.14 \\
\hline 4 & 187 & 9 & 21 & 10 & 20.78 & 18.70 & 8.90 \\
\hline 5 & 234 & 11 & 28 & 12 & 21.27 & 19.50 & 8.36 \\
\hline 6 & 352 & 16 & 32 & 17 & 22.00 & 20.71 & 11.00 \\
\hline MEDIA & 290.83 & 13 & 33.17 & 13.67 & 22.88 & 22.49 & 9.95 \\
\hline MEDIANA & 303.5 & 12 & 30 & 12 & 21.64 & 20.10 & 8.80 \\
\hline MODA & N/A & N/A & 21 & 12 & N/A & N/A & N/A \\
\hline $\mathrm{DE}$ & 69.27 & 3.85 & 13.26 & 4.93 & 4.50 & 6.76 & 2.79 \\
\hline
\end{tabular}

Nota: $S=$ oraciones, $C=$ cláusulas, $T=$ t-unidades, $M L S=$ palabras por oración, $M L T=$ palabras por t unidad T, $M L C=$ palabras por cláusula, $D E=$ desviación estándar, $N=$ tamaño de la muestra (ensayos)

Como se muestra en la Tabla 3, los ensayos producidos en colaboración fueron más fluidos con respecto al número total de palabras, oraciones, cláusulas, unidades T, palabras por oración (MLS), palabras por cláusula (MLC) y palabras por unidad $\mathrm{T}$ que el ensayo producido individualmente. Los estudiantes crearon ensayos más largos cuando trabajaron en grupo ya que porque compartieron sus ideas a través de la negociación, discusión y planificación con los miembros del equipo, ratificando lo que mencionaron Stein et al. (1997). 
Las palabras por oración (MLS), las palabras por cláusula (MLC) y las palabras por unidad T (MLT) también son más largas en los ensayos de colaboración, con relación a los ensayos escritos individualmente, lo que corrobora lo que Wolfe-Quintero et al. (1998), mencionó con respecto a la medida de la longitud de unidad de producción.

\section{Tabla 4}

\begin{tabular}{|c|c|c|c|c|c|c|c|}
\hline \multicolumn{8}{|c|}{ Longitud de Producción Grupo D } \\
\hline Ensayos & $\begin{array}{c}\text { No. de } \\
\text { Palabras }\end{array}$ & $\mathrm{S}$ & $\mathrm{C}$ & $\mathrm{T}$ & MLS & MLT & MLC \\
\hline \multicolumn{8}{|c|}{ Producción Colaborativa $(N=6)$} \\
\hline 1 & 708 & 29 & 84 & 37 & 24.41 & 19.14 & 8.43 \\
\hline 2 & 537 & 21 & 56 & 22 & 25.57 & 24.41 & 9.59 \\
\hline 3 & 675 & 28 & 72 & 28 & 24.11 & 24.11 & 9.38 \\
\hline 4 & 937 & 19 & 99 & 24 & 49.32 & 39.04 & 9.46 \\
\hline 5 & 720 & 19 & 85 & 19 & 37.89 & 37.89 & 8.47 \\
\hline 6 & 623 & 17 & 67 & 17 & 36.65 & 36.65 & 9.30 \\
\hline MEDIA & 700.00 & 22.17 & 77.17 & 24.50 & 32.99 & 30.21 & 9.10 \\
\hline MEDIANA & 691.50 & 20.00 & 78.00 & 23.00 & 31.11 & 30.53 & 9.34 \\
\hline MODA & N/A & 19.00 & N/A & N/A & N/A & N/A & N/A \\
\hline $\mathrm{DE}$ & 133.98 & 5.08 & 15.25 & 7.23 & 10.11 & 8.63 & 0.52 \\
\hline \multicolumn{8}{|c|}{ Producción Individual $(N=6)$} \\
\hline 1 & 194 & 7 & 15 & 9 & 27.71 & 21.56 & 12.93 \\
\hline 2 & 145 & 7 & 18 & 7 & 20.71 & 20.71 & 8.06 \\
\hline 3 & 236 & 9 & 17 & 12 & 26.22 & 19.67 & 13.88 \\
\hline 4 & 241 & 14 & 24 & 15 & 17.21 & 16.07 & 10.04 \\
\hline 5 & 151 & 2 & 16 & 9 & 75.50 & 16.78 & 9.44 \\
\hline 6 & 244 & 10 & 24 & 10 & 24.40 & 24.40 & 10.17 \\
\hline MEDIA & 201.83 & 8.17 & 19.00 & 10.33 & 31.96 & 19.86 & 10.75 \\
\hline MEDIANA & 215 & 8 & 17.5 & 9.5 & 25.31 & 20.19 & 10.10 \\
\hline MODA & N/A & 7 & 24 & 9 & N/A & N/A & N/A \\
\hline $\mathrm{DE}$ & 45.51 & 3.97 & 4.00 & 2.80 & 21.67 & 3.10 & 2.21 \\
\hline
\end{tabular}

Nota: $S=$ oraciones, $C=$ cláusulas, $T=$ t-unidades, $M L S=$ palabras por oración, $M L T=$ palabras por unidad T, $M L C=$ palabras por cláusula, $D E=$ desviación estándar, $N=$ tamaño de la muestra (ensayos)

Como se muestra en la Tabla 4, los ensayos producidos en colaboración fueron más fluidos con respecto al número total de palabras, oraciones, cláusulas, unidades T, palabras por oración (MLS) y palabras por unidad $\mathrm{T}$ que los ensayos producidos individualmente. Sin embargo, las palabras por cláusula (MLC) en los ensayos producidos individualmente fueron un poco más largas. Según Ortega (2003) y Henry (1996), las palabras 
por oración, palabra por cláusula y palabra por unidad T se usan para medir la complejidad sintáctica; esto significa que aquí los estudiantes fueron más fluidos escribiendo individualmente. Para resumir, diríamos que los estudiantes produjeron ensayos más largos cuando trabajaron escribiendo en grupo ya que compartieron más ideas a través de la negociación, discusión y planificación con los miembros del equipo como lo mencionaron Stein et al. (1997).

Las palabras por frase (MLS) y las palabras por unidad T (MLT) son más largas en los ensayos de colaboración, lo que corrobora lo que WolfeQuintero et al. (1998), mencionó con respecto a la medida de la longitud de unidad de producción.

Tabla 5

\begin{tabular}{|c|c|c|c|c|c|c|c|}
\hline \multicolumn{8}{|c|}{ Longitud de Producción Grupo E } \\
\hline Ensayos & $\begin{array}{c}\text { No. de } \\
\text { Palabras }\end{array}$ & $\mathrm{S}$ & $\mathrm{C}$ & $\mathrm{T}$ & MLS & MLT & MLC \\
\hline \multicolumn{8}{|c|}{ Producción Colaborativa $(N=6)$} \\
\hline 1 & 1039 & 55 & 113 & 54 & 18.89 & 19.24 & 9.19 \\
\hline 2 & 847 & 38 & 64 & 36 & 22.29 & 23.53 & 13.23 \\
\hline 3 & 873 & 47 & 82 & 46 & 18.57 & 18.98 & 10.65 \\
\hline 4 & 1003 & 47 & 102 & 39 & 21.34 & 25.72 & 9.83 \\
\hline 5 & 1017 & 38 & 106 & 44 & 26.76 & 23.11 & 9.59 \\
\hline 6 & 1051 & 41 & 122 & 47 & 25.63 & 22.36 & 8.61 \\
\hline MEDIA & 971.67 & 44.33 & 98.17 & 44.33 & 22.25 & 22.16 & 10.19 \\
\hline MEDIANA & 1010 & 44 & 104 & 45 & 21.81 & 22.74 & 9.71 \\
\hline MODA & N/A & 38 & N/A & N/A & N/A & N/A & N/A \\
\hline $\mathrm{DE}$ & 88.48 & 6.62 & 21.41 & 6.35 & 3.39 & 2.61 & 1.64 \\
\hline \multicolumn{8}{|c|}{ Producción Individual $(N=6)$} \\
\hline 1 & 192 & 4 & 12 & 3 & 48 & 64 & 16 \\
\hline 2 & 266 & 20 & 22 & 16 & 13.30 & 16.63 & 12.09 \\
\hline 3 & 191 & 12 & 13 & 10 & 15.92 & 19.10 & 14.69 \\
\hline 4 & 190 & 6 & 15 & 5 & 31.67 & 38.00 & 12.67 \\
\hline 5 & 171 & 3 & 14 & 8 & 57 & 21.38 & 12.21 \\
\hline 6 & 156 & 4 & 16 & 7 & 39 & 22.29 & 9.75 \\
\hline MEDIA & 194.33 & 8.17 & 15.33 & 8.17 & 34.15 & 30.23 & 12.90 \\
\hline MEDIANA & 190.5 & 5 & 14.5 & 7.5 & 35.33 & 21.83 & 12.44 \\
\hline MODA & N/A & 4 & N/A & N/A & N/A & N/A & N/A \\
\hline $\mathrm{DE}$ & 37.91 & 6.65 & 3.56 & 4.54 & 17.38 & 18.17 & 2.19 \\
\hline
\end{tabular}

Nota: $S=$ oraciones, $C=$ cláusulas, $T=$ t-unidades, $M L S=$ palabras por oración, $M L T=$ palabras por unidad T, $M L C=$ palabras por cláusula, $D E=$ desviación estándar, $N=$ tamaño de la muestra (ensayos) 
Como se muestra en la Tabla 5, los ensayos producidos en colaboración fueron más fluidos con respecto al número total de palabras, oraciones, cláusulas y unidades T. Sin embargo, las palabras por oración (MLS), las palabras por unidad de T (MLT) y las palabras por cláusula (MLC) en cada producción individual fueron más largas que los ensayos escritos en colaboración. Según Ortega (2003) y Henry (1996), las palabras por oración (MLS), la palabra por cláusula (MLC) y la palabra por unidad T (MLT) se utilizan para medir la complejidad sintáctica.

Tabla 6

\begin{tabular}{|c|c|c|c|c|c|c|c|}
\hline \multicolumn{8}{|c|}{ Longitud de Producción Grupo F } \\
\hline Ensayos & $\begin{array}{c}\text { No. de } \\
\text { Palabras }\end{array}$ & S & C & $\mathrm{T}$ & MLS & MLT & MLC \\
\hline \multicolumn{8}{|c|}{ Producción Colaborativa $(N=6)$} \\
\hline 1 & 1227 & 32 & 91 & 34 & 38.34 & 36.09 & 13.48 \\
\hline 2 & 924 & 39 & 83 & 41 & 23.69 & 22.54 & 11.13 \\
\hline 3 & 795 & 23 & 78 & 24 & 34.57 & 33.13 & 10.19 \\
\hline 4 & 724 & 16 & 89 & 23 & 45.25 & 31.48 & 8.13 \\
\hline 5 & 836 & 24 & 79 & 29 & 34.83 & 28.83 & 10.58 \\
\hline 6 & 767 & 29 & 97 & 29 & 26.45 & 26.45 & 7.91 \\
\hline MEDIA & 878.83 & 27.17 & 86.17 & 30 & 33.86 & 29.75 & 10.24 \\
\hline MEDIANA & 815.50 & 26.5 & 86 & 29 & 34.70 & 30.15 & 10.39 \\
\hline MODA & N/A & $\mathrm{N} / \mathrm{A}$ & N/A & 29 & N/A & $\mathrm{N} / \mathrm{A}$ & $\mathrm{N} / \mathrm{A}$ \\
\hline $\mathrm{DE}$ & 183.63 & 7.99 & 7.44 & 6.69 & 7.87 & 4.86 & 2.06 \\
\hline \multicolumn{8}{|c|}{ Producción Individual $(N=6)$} \\
\hline 1 & 401 & 11 & 27 & 12 & 36.45 & 33.42 & 14.85 \\
\hline 2 & 331 & 11 & 29 & 12 & 30.09 & 27.58 & 11.41 \\
\hline 3 & 202 & 9 & 19 & 12 & 22.44 & 16.83 & 10.63 \\
\hline 4 & 306 & 5 & 29 & 7 & 61.20 & 43.71 & 10.55 \\
\hline 5 & 204 & 12 & 22 & 11 & 17 & 18.55 & 9.27 \\
\hline 6 & 176 & 5 & 15 & 4 & 35.20 & 44 & 11.73 \\
\hline MEDIA & 270 & 8.83 & 23.50 & 9.67 & 33.73 & 30.68 & 11.41 \\
\hline MEDIANA & 255 & 10 & 24.5 & 11.5 & 32.65 & 30.50 & 11.02 \\
\hline MODA & N/A & 11 & 29 & 12 & N/A & N/A & N/A \\
\hline $\mathrm{DE}$ & 89.44 & 3.13 & 5.79 & 3.39 & 15.39 & 11.86 & 1.89 \\
\hline
\end{tabular}

Nota: $S=$ oraciones, $C=$ cláusulas, $T=$ t-unidades, $M L S=$ palabras por oración, $M L T=$ palabras por unidad T, $M L C=$ palabras por cláusula, $D E=$ desviación estándar, $N=$ tamaño de la muestra (ensayos)

Como se muestra en la Tabla 6, los ensayos producidos en grupo fueron más fluidos con respecto al número total de palabras, oraciones, cláusulas, unidades $\mathrm{T}$ y palabras por oración (MLS) que los ensayos producidos individualmente. Sin embargo, las palabras por cláusula (MLC) y las palabras por unidad T (MLT) fueron ligeramente más largas en los ensayos escritos individualmente. De acuerdo con Ortega (2003) y Henry (1996), las 
palabras por oración (MLS), palabra por cláusula (MLC) y palabra por unidad de T (MLT) se utilizan para medir la complejidad sintáctica. Así diríamos que los estudiantes parecen escribir un mayor número de palabras en sus ensayos cuando escriben en grupo, porque generan más ideas con otros miembros del grupo a través de la negociación, discusión y planificación con los miembros del equipo, corroborando lo que mencionaron Stein et al. (1997).

Tabla 7 Beneficios al utilizar herramientas colaborativas

\begin{tabular}{|c|c|}
\hline $\begin{array}{l}\text { Métodos de } \\
\text { colección de datos } \\
\text { (N=33) }\end{array}$ & \multicolumn{1}{|c|}{ Beneficios } \\
\hline \multicolumn{2}{|c|}{ Beneficios sociales al utilizar una herramienta colaborativa } \\
\hline Cuestionario & $\begin{array}{l}\text { El 91.9\% de los estudiantes estuvieron de acuerdo con el hecho de } \\
\text { que durante la escritura colaborativa su interacción aumentó con } \\
\text { otros miembros. }\end{array}$ \\
\hline \multicolumn{3}{|c|}{ Beneficios psicológicos de la escritura colaborativa } \\
\hline Cuestionario & $\begin{array}{l}\text { Un total de 85.9\% de los estudiantes estuvieron de acuerdo en que la } \\
\text { escritura colaborativa aumentó su motivación para escribir. }\end{array}$ \\
\hline \multicolumn{3}{|c|}{ Beneficios académicos de la escritura colaborativa } \\
\hline Cuestionario & $\begin{array}{l}\text { Un total de 85.9\% de los estudiantes estuvieron de acuerdo en que } \\
\text { se esforzaron más durante el proceso de escritura colaborativa para } \\
\text { que otros miembros del grupo puedan ver su trabajo. }\end{array}$ \\
\hline Cuestionario & $\begin{array}{l}\text { El 82.8\% de los estudiantes aceptaron sugerencias de sus } \\
\text { compañeros para corregir sus composiciones. }\end{array}$ \\
\hline \multicolumn{1}{|c|}{ Interacción entre profesor y alumnos } \\
\hline Cuestionario & $\begin{array}{l}\text { El 76.8\% de los estudiantes estuvieron de acuerdo en que } \\
\text { aprendieron más al interactuar con sus compañeros que con el } \\
\text { maestro. }\end{array}$ \\
\hline
\end{tabular}

\section{Discusión y Conclusion}

Haciendo referencia a los resultados obtenidos en los ensayos escritos colaborativa e individualmente, podemos ver que no existe mucha variación en los valores de la media de MLS, MLT y MLC. Así, por ejemplo en el grupo A, tabla 1, los valores de la media en MLS, MLT en los ensayos individuales son un poco mayores. Si hacemos referencia a lo que mencionó WolfeQuintero et al. (1998), con relación a la medida de la longitud de unidad de producción, en este caso no aplicaría ya que para que se cumpla esto los tres valores MLS, MLT y MLC en la media tendrían que ser mayores. Sin embargo, considerando lo que Stein et al. (1997) mencionaron al decir que los estudiantes tienen un nivel más alto cuando hay un incremento en el número de palabras, se puede decir que en este caso los estudiantes del grupo A son más fluidos ya que el valor de la media es de 814.33 palabras comparado al valor de escritura individual que es de 228.

Tomando como ejemplo el Grupo B, y si tomamos los tres valores MLS, MLT y MLC de la media en los ensayos colaborativos, podemos ver 
que estos son más altos que los valores de los ensayos escritos individuamente. En este caso se puede decir que los estudiantes del grupo B que escribieron utilizando una herramienta colaborativa fueron más fluidos que los estudiantes que escribieron individualmente ya que tanto el valor del número total de palabras como los tres valores MLS, MLT y MLC mencionados anteriormente son más altos.

De la misma forma, tomando como ejemplo el grupo E, podemos decir que aquí los estudiantes que escribieron individualmente son más fluidos si tomamos como referencia los valores MLS, MLT y MLC. Sin embargo, si se toma como referencia el número total de palabras, este no sería el caso ya que este valor es menor al número total de palabras escritas en colaboración.

Los estudiantes que escribieron colaborativa e individualmente tuvieron 90 minutos para escribir sus ensayos, sin embargo, los estudiantes que escribieron solos tuvieron un mayor grado de concentración y su responsabilidad de hacer un buen ensayo fue más grande ya que no interactuaban con otros estudiantes. De igual forma, a los estudiantes que escribieron colaborativamente les tomo más tiempo organizarse para empezar a escribir el ensayo, por lo que este tipo de actividad ayudó a cada estudiante a tomar responsabilidad y concentrarse en la tarea asignada, ya que el trabajo de escribir el ensayo fue compartida por todos los miembros del grupo. Es ompirtante también destacar que ambos grupos tenían acceso al diccionario lo que les permitió redactar de una mejor manera sus ensayos; caso contrario la redacción hubiese sido más complicada para ellos.

De acuerdo a los resultados obtenidos en esta investigación se puede decir que la mayoría de los estudiantes prefieren escribir en grupo en lugar de escribir individualmente. Esto se debe a que los estudiantes se sintieron apoyados por sus compañeros, y para muchos estudiantes tímidos se les hizo más fácil comunicarse y dar sus ideas de una manera más cómoda a través de Google Docs. Los estudiantes se mostraron satisfechos con la efectividad de Google Docs para trabajar en grupo ya que los estudiantes escribieron sus composiciones de una manera muy fácil tomando en cuenta que al estar conectados a internet tuvieron ayuda inmediata.

Se concluye que los estudiantes produjeron ensayos más largos cuando escribieron colaborativamente porque tuvieron más ideas a través de la negociación, discusión y planificación con los miembros del equipo, corroborando lo que mencionaron Stein et al. (1997). Por esta razón, esta investigación debería servir como base para futuras investigaciones.

Durante el proceso de aprendizaje de inglés, sabemos como maestros que una de las habilidades más difíciles para los estudiantes es la escritura. Por lo tanto, es importante que los profesores introduzcan actividades de escritura colaborativa utilizando herramientas tecnológicas ya que estas permiten a varios alumnos escribir un solo documento en tiempo real. 
Los profesores deberían considerar los métodos sugeridos anteriormente para evaluar la fluidez de escritura de sus estudiantes dependiendo de qué se desee medir, sea este el número total de palabras o la complejidad de las oraciones. Estos resultados ayudan al profesor a tomar decisiones en su clase realizando más actividades de escritura orientadas a mejorar el nivel de de producción escrita en inglés de sus estudiantes.

\section{Recomendaciones}

La medición de la fluidez con respecto al número total de palabras es un método que beneficia tanto al maestro como al alumno. Con este método el profesor podría tener una idea más clara del nivel de escritura de sus estudiantes, en un tiempo más corto, al momento de evaluar el nivel de los estudiantes al inicio de un nuevo semestre. Es recomendable que los maestros implementen actividades de escritura colaborativa en las aulas ya que los estudiantes se beneficiarían de todas las ventajas de este tipo de actividad, y de esta manera se sentirían más motivados.

\section{References:}

1. Henry, K. (1996). Early L2 Writing Development: A Study of Autobiographical Essays by University-Level Students of Russian. The MODArn Language Journal, 309-326.

2. La Guía Nacional de Currículo en inglés (2012, Septiembre 14). Ministerio de Educación. Retrieved Diciembre 01, 2018, from Currículo fortalecimiento del inglés:

3. Larsen-Freeman, D. (1978). An ESL Index of Development. TESOL Quarterly, 439.

4. Lu, X. (2010). Automatic analysis of syntactic complexity in second language writing. International Journal of Corpus Linguistics, 474496.

5. Storch, N. (2011). Collaborative Writing in L2 Context:Processes, Outcomes, and Future Directions. Anual Review of Applied Linguistics, 273-285.

6. Wolfe-Quintero, K., Inagaki, S., \& kim, H.-Y. (1998). Second Language Development in Writing:Measures of Fluency, Accuracy and Complexity. Manoa: Richard Schmidt.

7. Stein, N. L., Bernas, R. S., \& Calicchia, D. (1997). Conflict talk: Understanding and resolving arguments. In M. Noonan \& T. Givón (Eds.), Conversation: Cognitive, Communicative and social perspectives. Typological studies in language, Vol. 34 (pp. 233-267). Amsterdam, NL: John Benjamins Publishing. 